\title{
Increased random exploration in schizophrenia is associated
} with inflammation

\author{
Flurin Cathomas (iD ${ }^{1,2 凶}$, Federica Klaus (iD ${ }^{1,3}$, Karoline Guetter ${ }^{1}$, Hui-Kuan Chung (D) $^{4}$, Anjali Raja Beharelle $e^{4,5}$, Tobias R. Spiller (iD ${ }^{6}$, \\ Rebecca Schlegel ${ }^{1}$, Erich Seifritz ${ }^{1,5,7}$, Matthias N. Hartmann-Riemer ${ }^{1}$, Philippe N. Tobler ${ }^{4,5,7}$ and Stefan Kaiser ${ }^{8}$
}

One aspect of goal-directed behavior, which is known to be impaired in patients with schizophrenia (SZ), is balancing between exploiting a familiar choice with known reward value and exploring a lesser known, but potentially more rewarding option. Despite its relevance to several symptom domains of SZ, this has received little attention in SZ research. In addition, while there is increasing evidence that SZ is associated with chronic low-grade inflammation, few studies have investigated how this relates to specific behaviors, such as balancing exploration and exploitation. We therefore assessed behaviors underlying the exploration-exploitation trade-off using a three-armed bandit task in 45 patients with SZ and 19 healthy controls (HC). This task allowed us to dissociate goal-unrelated (random) from goal-related (directed) exploration and correlate them with psychopathological symptoms. Moreover, we assessed a broad range of inflammatory proteins in the blood and related them to bandit task behavior. We found that, compared to HC, patients with SZ showed reduced task performance. This impairment was due to a shift from exploitation to random exploration, which was associated with symptoms of disorganization. Relative to HC, patients with SZ showed a pro-inflammatory blood profile. Furthermore, high-sensitivity C-reactive protein (hsCRP) positively correlated with random exploration, but not with directed exploration or exploitation. In conclusion, we show that low-grade inflammation in patients with SZ is associated with random exploration, which can be considered a behavioral marker for disorganization. hsCRP may constitute a marker for severity of, and a potential treatment target for maladaptive exploratory behaviors.

npj Schizophrenia (2021)7:6; https://doi.org/10.1038/s41537-020-00133-0

\section{INTRODUCTION}

Schizophrenia (SZ) is a complex neuropsychiatric disorder characterized by a wide range of symptoms ${ }^{1}$. Negative symptoms and disorganization are strongly associated with reduced social and occupational functioning, and are challenging to treat ${ }^{2-5}$. Therefore, understanding the shared and dissociable mechanisms underlying these symptoms is crucial in increasing our knowledge about the etio-pathophysiology of the disorder and the much needed development of novel treatments.

Negative symptoms and disorganization have been linked to impairments of goal-directed behaviors ${ }^{6,7}$. Investigating behaviors relevant to these symptom dimensions in SZ with objective, taskbased measures that allow the assessment of complex, welldefined behaviors with ecological validity is crucial $^{8,9}$. The exploration-exploitation trade-off concerns the decision between exploiting a familiar option with known reward value and exploring a lesser known option with more uncertain, but potentially higher reward value. This trade-off may constitute a paradigmatic case for assessing goal-directed behaviors, which have been shown to be impaired in patients with $S Z^{10,11}$. To study the exploration-exploitation trade-off in a controlled experimental setting, a human computerized task, where the participant has to repeatedly choose between different "slot machines" (bandits) that gradually change their reward values has been developed $^{12,13}$. Accordingly, participants have to decide when to switch from exploitative to exploratory strategies when aiming to obtain the highest total reward pay-off possible ${ }^{13,14}$. An optimal balance between exploration and exploitation results in a maximization of the obtained reward, and fulfils the fundamental need of foraging organisms to adapt to a complex and changing world (for review ref. ${ }^{15}$ ).

Few studies have applied exploration-exploitation tasks in patients with SZ and no firm conclusions can be drawn so far: Strauss and colleagues found that patients with SZ show decreased uncertainty-driven directed exploration, which was associated with clinically assessed negative symptoms ${ }^{16}$. Another study reported that patients with SZ displayed increased noveltyseeking behaviors ${ }^{17}$. In addition, while several brain regions (midbrain and prefrontal areas) and neurotransmitters (dopamine, noradrenaline, and acetylcholine) have been implicated in mediating exploration-exploitation trade-offs ${ }^{10,15,18,19}$, the underlying etiological mechanisms remain to be elucidated.

Inflammation has been suggested to be a key mechanism for disturbances of motivation and goal-directed behavior ${ }^{20,21}$. Preclinical animal models and human studies have shown a close interaction between the peripheral immune system, and the central nervous system in health and the development of neuropsychiatric disorders ${ }^{22,23}$. Indeed, several studies have reported that patients with SZ display alterations in immune function indexed by cytokines, chemokines, and acute-phase

\footnotetext{
${ }^{1}$ Department of Psychiatry, Psychotherapy and Psychosomatics, Psychiatric Hospital, University of Zurich, 8032 Zurich, Switzerland. ${ }^{2}$ Fishberg Department of Neuroscience, Friedman Brain Institute, Icahn School of Medicine at Mount Sinai, New York, USA. ${ }^{3}$ Department of Psychiatry, University of California San Diego, San Diego, USA. ${ }^{4}$ Zurich Center for Neuroeconomics, Department of Economics, University of Zurich, 8006 Zurich, Switzerland. ${ }^{5}$ Neuroscience Center Zurich, ETH Zurich and University of Zurich, 8057 Zurich, Switzerland. ${ }^{6}$ University of Zurich, University Hospital Zurich, Department of Consultation-Liaison Psychiatry and Psychosomatic Medicine, Ramistrasse 100, 8091 Zurich, Switzerland. ${ }^{7}$ Zurich Center for Integrative Human Physiology, University of Zurich, 8057 Zurich, Switzerland. ${ }^{8}$ Division of Adult Psychiatry, Department of Psychiatry, Geneva University Hospitals, Chemin du Petit-Bel-Air, 1225 Chêne-Bourg, Switzerland. ${ }^{凶}$ email: flurin.cathomas@puk.zh.ch
} 
proteins in both blood and cerebral spinal fluid, which are indicative of chronic low-grade inflammation (see ref. ${ }^{24}$ for metaanalysis).

Recent studies have started to investigate whether these immune changes correlate with specific behaviors ${ }^{25}$. With regard to exploration and exploitation, the concept of sickness behavior as an adaptive behavioral response to acute infections is of high relevance ${ }^{26,27}$. Sickness behavior can be interpreted as a shortterm disturbance of exploratory behaviors that could have beneficial energy-saving effects by shifting energy resources toward the immune system to facilitate clearing infections and wound healing. However, in our modern world with fewer infectious challenges and better sanitation, chronic low-grade inflammation might be maladaptive leading to inefficient performance $^{28}$. To our knowledge, no study has so far investigated the relationship of a pro-inflammatory state with an objective task assessment of exploration-exploitation behavior, despite the high relevance for several symptom domains of SZ. Overall, we hypothesized that patients with SZ will display reduced task outcome based on impairments in balancing between exploration and exploitation behaviors, and that the underlying behavioral impairments are associated with a lowgrade, pro-inflammatory state.

Therefore, we first assessed negative, disorganized, and cognitive symptoms in clinically and pharmacologically stable patients with SZ and healthy controls ( $\mathrm{HC}$ ). We then examined exploration vs. exploitation behaviors using a three-armed computerized bandit task, and investigated correlations between overall task performance, directed, and random exploration and exploitation with psychopathological measures. Next, we assessed group differences (SZ vs. HC) in a broad array of cytokines and chemokines in the blood. To investigate and visualize the complex interaction between the individual inflammatory parameters and their relationship with group affiliation (SZ or $\mathrm{HC}$ ), we used network analysis. Lastly, we investigated the relationships between the immune markers that were different between groups and task-based behaviors.

\section{RESULTS}

\section{Sociodemographic data}

Sociodemographic and clinical data of the sample are summarized in Table 1. Our SZ sample showed a wide range of negative and disorganized symptoms, and few positive symptoms. Patients with SZ completed fewer years of formal education $(p=0.003)$ and had a higher body mass index (BMl; $p=0.009$ ) than HC. Nine participants were prescribed clozapine and only one patient used typical antipsychotics. Three patients used lorazepam (up to $1 \mathrm{mg} /$ d) and five patients took antidepressants. The SZ group displayed a significantly lower score in Personal and Social Performance Scale (PSP) as a measure of global level of functioning $(p<0.001)$. Patients with SZ also displayed a lower cognitive score $(p<0.001)$.

\section{Patients with SZ display impaired performance in the exploration-exploitation bandit task}

First, the total reward earned from the bandit task was calculated for each group. Patients with SZ obtained significantly fewer points than HC (HC: $62.9 \pm 5.0$; SZ: $59.1 \pm 4.7), t=2.89 p=0.005$, $d=0.78$; Fig. $1 \mathrm{~A})$. Both $\mathrm{HC}$ and patients with $\mathrm{SZ}$ earned significantly more than the simulated random chooser (random choosers: 51.9, HC: $p<0.0001$, SZ: $p<0.0001$ ). Thus, even though patients with SZ earned less than HC, individuals with SZ clearly performed better than chance.

In a next step, we aimed to investigate potential reasons for why patients with SZ earned less reward than HC. To do so, we analyzed exploration and exploitation behaviors. We found that exploitation, defined as the continuous selection of the best bandit option, was reduced in patients with SZ (HC: $5.71 \pm 2.3$; SZ: $4.65 \pm 1.5), t=2.14, p=0.036, d=0.55$; Fig. 1B). In contrast, there were no significant differences between groups in the length of consecutively selecting other options $\left(p^{\prime} s>0.7\right)$. We then tested whether there were group differences in exploratory behaviors. SZ patients showed more random exploration $(\mathrm{HC}=23.32 \pm 13.3, \mathrm{SZ}$ $=35.63 \pm 23.1 ; U=275.5, p=0.042, d=0.65$; Fig. $1 C)$, but similar levels of directed exploration $(H C=13.6 \pm 6.7, S Z=19.6 \pm 13.7$; $U=302, p=0.104$; Fig. 1D). Furthermore, there was a significant negative correlation between random exploration and points earned in the task $(r(p)=-0.34, p=0.025)$ in patients with SZ.

Next, we performed correlational analyses between the task parameters and psychopathological domains relevant to SZ: negative and depressive symptoms, symptoms of disorganization, and cognitive symptoms. Interestingly, symptoms of disorganization, assessed by the Positive and Negative Syndrome Scale (PANSS) disorganized factor, correlated positively with random exploration $(r(s)=0.31, p=0.045)$ and negatively with exploitation $(r(s)=-0.36, p=0.018)$, the two task parameters that contributed to the decreased overall task performance in SZ patients compared to $\mathrm{HC}$, but not with directed exploration $(r(s)$ $=0.27, p=0.078)$. While we observed a significant negative correlation between the PANSS disorganized factor and composite cognitive score $(r(s)=-0.33, p=0.029)$, task parameters did not correlate with cognitive, positive, negative, or depressive symptoms (Supplementary Table 1). Since antipsychotic medications are known to have an influence on various behaviors and cognition, we correlated chlorpromazine equivalents with task parameters, but did not observe a significant correlation (Supplementary Table 1).

Together, our data show that patients with SZ display impaired task performance as they earned fewer points in the bandit task. Analyzing the underlying behaviors revealed that this deficit is due to increased random exploration and decreased exploitation, which both correlated with the PANSS disorganized factor.

\section{Patients with SZ show a pro-inflammatory phenotype}

To investigate group differences in blood immune profiles between SZ and HC, we used two different exploratory strategies. First, we investigated group differences of individual immune parameters between $\mathrm{HC}$ and patients with SZ. Several immune markers differed between groups, indicative of a pro-inflammatory phenotype in patients with SZ compared to $\mathrm{HC}$ (Fig. 2A and Supplementary Table 3): high-sensitivity C-reactive protein (hsCRP; $U=243.5, \quad p=0.007, d=0.96)$, interleukin (IL)-6 $(t=-2.31$, $p=0.025, d=0.74)$, tumor necrosis factor (TNF)-related apoptosisinducing ligand (TRAIL; $t=-4.16, p<0.001, d=1.05$ ), TNF-related activation-induced cytokine TRANCE (TRANCE; $t=-2.09, p=0.041$, $d=0.59$ ), fibroblast growth factor 21 (FGF-21; $t=-2.19, p=0.033$, $d=0.61)$, chemokine ligand (CCL) $2(t=-2.05, p=0.045, d=0.61)$, $\operatorname{CCL} 7(t=-2.23, p=0.030, d=0.71), \operatorname{CCL} 11(t=-2.74, p=0.008$, $d=0.83)$, and $\operatorname{CCL} 20(U=158.5, p<0.001, d=1.02)$ were increased in the SZ group, while stem cell factor $(t=2.13, p=0.038, d=0.62)$, CXCL11 $(U=223, p=0.026, d=0.56)$, and CCL28 $(U=183.5$, $p=0.003, d=0.48)$ were decreased.

hsCRP $(p=0.022)$, TRAIL $(p=0.001), \operatorname{CCL} 20(p=0.003), \mathrm{CCL} 7$ $(p=0.027)$, and CCL11 $(p=0.010)$ remained significantly different after controlling for age, sex, and BMI.

We then performed network analysis of the inflammatory markers that were significantly different between the two groups. Given that cytokines and other pro-inflammatory markers are known to influence each other reciprocally, network analysis can reveal distinct associations between group affiliation and the included proteins. Controlling for the associations with all other variables in the network, we found group affiliation to be uniquely correlated with several inflammatory markers, most strongly with CCL20, TRAIL, and hsCRP (Fig. 2B and Supplementary Figs. 3-7). 
Table 1. Sample characteristics.

\begin{tabular}{|c|c|c|c|c|}
\hline & $\mathrm{HC}(n=19)$ & $\mathrm{SZ}(n=45)$ & \multicolumn{2}{|c|}{ Test statistics } \\
\hline Age (years) & $32.53(9.45)$ & $34.00(10.47)$ & $t=-0.53$ & 0.60 \\
\hline Formal education (years) ${ }^{a}$ & $14.39(2.23)$ & $12.26(3.88)$ & $U=227$ & 0.003 \\
\hline Number of hospitalizations & - & $5.42(5.44)$ & - & - \\
\hline Number of psychotic episodes & - & $5.31(5.14)$ & - & - \\
\hline Illness onset (age, years) & - & $23.91(6.88)$ & - & - \\
\hline Illness duration (months) & - & $121.07(101.28)$ & - & - \\
\hline Chlorpromazine equivalents (mg/day) & - & $514.67(444.82)$ & - & - \\
\hline Clozapine (yes/no) & - & $9 / 36$ & - & - \\
\hline Body mass index & $23.40(4.32)$ & $26.40(4.66)$ & $U=250$ & 0.009 \\
\hline \multicolumn{5}{|l|}{ Psychopathology } \\
\hline PANSS negative factor ${ }^{b}$ & - & $15.49(6.82)$ & - & - \\
\hline PANSS positive factor ${ }^{c}$ & - & $5.96(2.70)$ & - & - \\
\hline PANSS disorganized factor ${ }^{d}$ & - & $5.87(2.48)$ & - & - \\
\hline PANSS depressive factor ${ }^{\mathrm{e}}$ & - & $5.33(2.45)$ & - & - \\
\hline \multicolumn{5}{|l|}{ Cognition } \\
\hline Letter-Number Span Test & $20.16(2.06)$ & $18.84(1.93)$ & $t=2.44$ & 0.018 \\
\hline Symbol Coding Test & $81.94(18.75)$ & $59.43(13.28)$ & $t=4.62$ & $<0.001$ \\
\hline Cognitive score $\mathrm{f}^{\mathrm{f}}$ & $0(0.68)$ & $-0.88(0.70)$ & $t=4.53$ & $<0.001$ \\
\hline \multicolumn{5}{|l|}{ Functioning } \\
\hline
\end{tabular}

Taken together, several pro-inflammatory cytokines and chemokines were elevated in patients with SZ compared to $\mathrm{HC}$, indicative of a pro-inflammatory state. Across all the analyses, hsCRP, CCL20, and TRAIL were most strongly associated with group.

\section{Random but not directed exploration is associated with increased hsCRP}

Finally, we correlated bandit task behaviors with TRAIL, CCL20, and hsCRP, the immune parameters that were most strongly associated with group across the analyses, in patients with SZ. To control for multiple comparisons, significance level was set at $p<0.017$ (0.05/3 parameters).

We found a significant positive correlation of hsCRP with random exploration $(r(s)=0.40, p=0.008$; Fig. $3 \mathrm{~A})$, but not exploitation $(r(s)=-0.27, p=0.081$; Fig. $3 \mathrm{~B})$, or directed exploration $(r(s)=0.17, p=0.284$; Fig. 3C). The correlation between random exploration and hsCRP remained significant after controlling for age, BMI, sex, smoking, cognition, chlorpromazine equivalents, PSP, years of formal education, number of hospitalizations, number of psychotic episodes, illness duration, and age at illness onset $(r=0.40, p=0.026)$. There were no correlations of CCL20 or TRAIL with task parameters (Supplementary Table 4).

\section{DISCUSSION}

In the present study, we investigated the link between shifted balancing of exploration vs. exploitation using an objective taskbased measure, and inflammatory parameters in patients with SZ. Compared to HC, patients with SZ showed reduced performance in the bandit task. Furthermore, we demonstrated that reduced task performance was associated with increased random exploration in SZ. Random exploration correlated with the acute-phase protein hsCRP.

To our knowledge, the specific increase in random exploration associated with reduced overall performance has not been previously reported for patients with SZ. However, in the study of Martinelli and colleagues, patients with SZ showed increased novelty seeking on a different version of the three-armed bandit 
A)

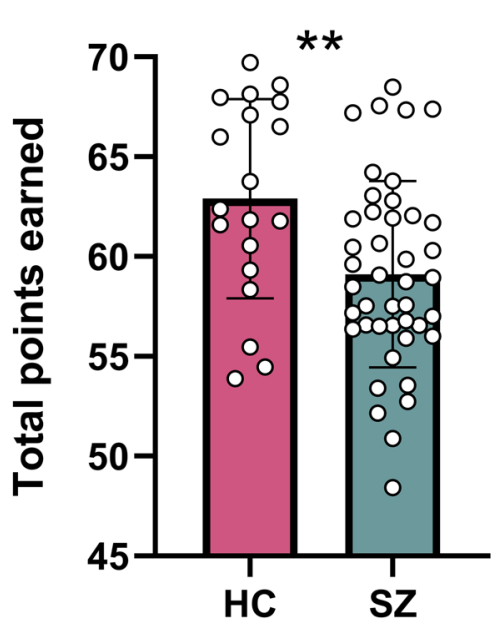

C)

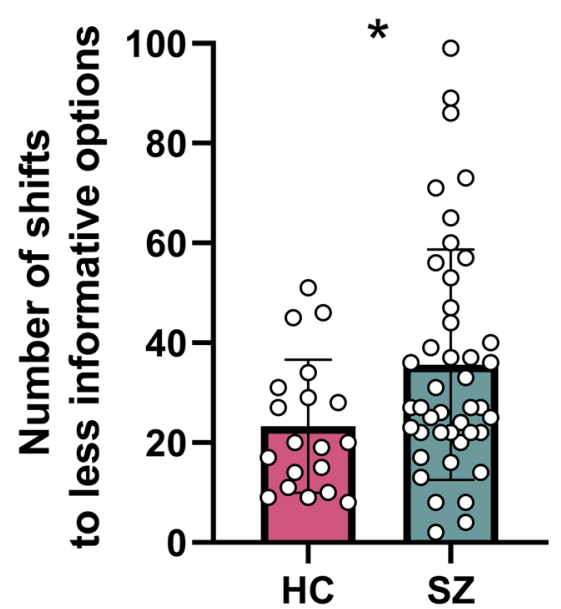

B)
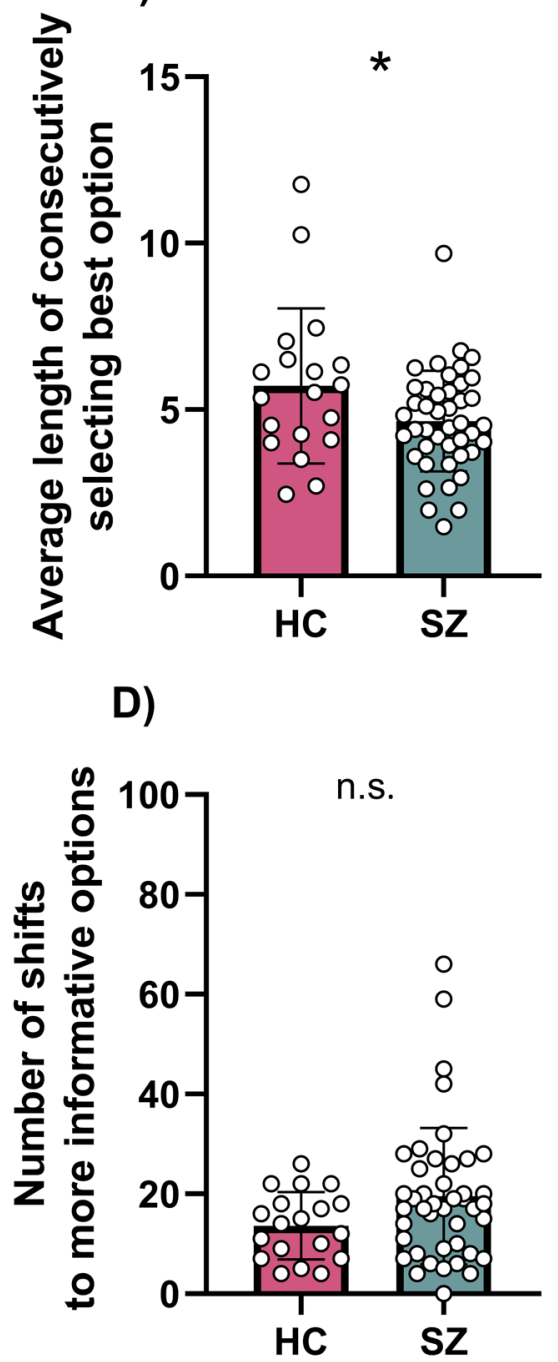

Fig. 1 Bandit task performance in patients with schizophrenia compared to healthy controls. A Patients with SZ earned fewer points in a three-armed bandit task than $\mathrm{HC}$. Compared to $\mathrm{HC}$, patients with SZ patients with SZ displayed B reduced exploitation of the best option, and showed $\mathbf{C}$ more random exploration, but $\mathbf{D}$ little differences in directed exploration. ${ }^{*} p<0.05,{ }^{* *} p<0.01$. n.s. non significant.

task $^{17}$. Although the task and the analysis are not directly comparable, their findings are consistent with ours as novelty seeking and random exploration tap into similar functions. In addition, several studies using simpler tasks have shown that patients with SZ display impaired ability to keep their best decisions and instead more often show "win-shift" behaviors, which is in line with our finding of reduced exploitation ${ }^{29,30}$. In contrast to findings from our study, Strauss and colleagues showed, using a "temporal utility integration task" that, compared to $\mathrm{HC}$, patients with SZ have decreased uncertainty-driven directed exploration, an effect that was most pronounced in patients with SZ with high levels of negative symptoms ${ }^{16}$. Further, directed exploration was negatively associated with anhedonia in $\mathrm{SZ}^{16}$. Although the use of different tasks makes direct comparisons difficult, it is interesting that in the present study patients with SZ did not show differences in directed exploration or correlations of negative symptoms with task parameters.

Instead, we observed a positive correlation between symptoms of disorganization and random exploration. Compared to other symptom domains, symptoms of disorganization have been the least studied, but are closely linked to functional outcomes ${ }^{2,31}$. Specifically, they have been associated with lack of insight ${ }^{32}$, lower quality of life ${ }^{33}$, and impaired long-term functioning ${ }^{34}$. In addition, several studies have shown that symptoms of disorganization are related to symptoms of cognition ${ }^{31,35}$. In line with these findings, we show a negative correlation between PANSS disorganized factor and cognitive score. However, the increased random exploration cannot be solely explained by cognition because we did not find a correlation between cognitive score and task behaviors. Taken together, our data suggest that exploratory behaviors assessed by the bandit task are less relevant to negative symptoms, but are more related to symptoms of disorganization, i.e., impairment of integrating complex inputs about a changing environment into an organized, efficient process.

The neural mechanisms underlying exploration-exploitation trade-offs and random exploration in particular have not been fully elucidated yet. Several neurotransmitters, including dopamine, acetylcholine, and norepinephrine have been suggested to modulate exploration-exploitation trade-offs ${ }^{10}$. While the neuromodulator dopamine has been consistently implicated in balancing exploration with exploitation ${ }^{36-38}$, the underlying etiological causes of this dopamine dysregulation are not well understood. There is a growing body of evidence indicating SZ is associated with chronic low-grade inflammation ${ }^{25,39,40}$ and that pro-inflammatory cytokines 


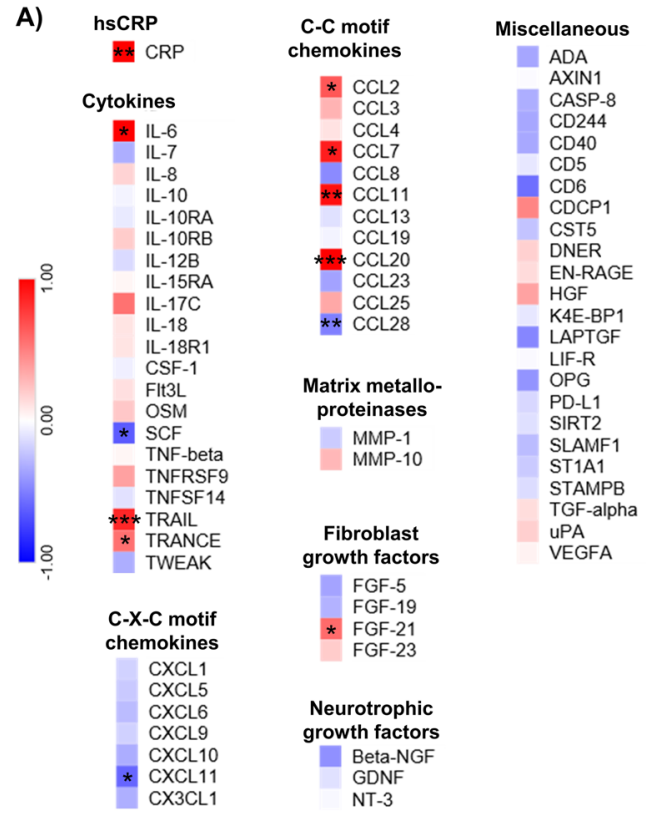

B)

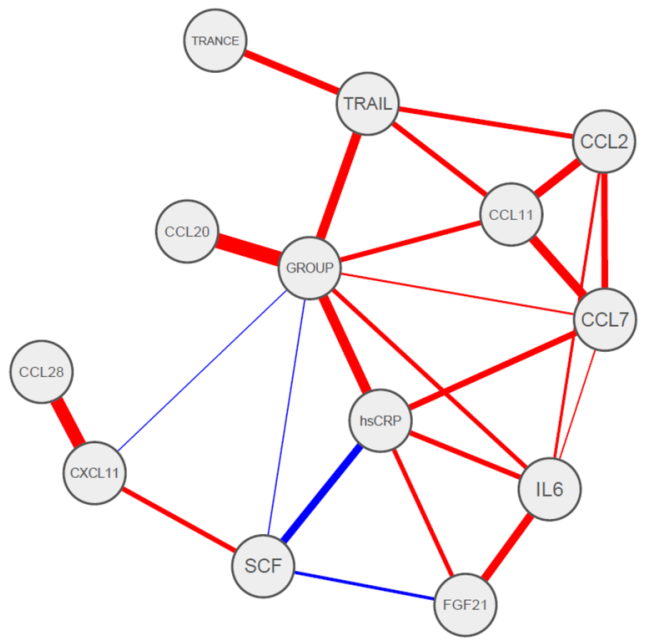

Fig. 2 Peripheral blood inflammatory markers. A Heat maps of standardized $z$-scores of inflammatory markers. Red indicates increased expression and blue decreased expression (patients with schizophrenia vs. healthy control subjects). B Network analysis. The thickness of an edge corresponds to its weight. Lines indicate positive (red) or negative (blue) partial correlation between two nodes. ${ }^{*} p<0.05,{ }^{* *} p<0.01$, ${ }^{* * *} p<0.001$. For a list of abbreviations see Supplementary Table 2.
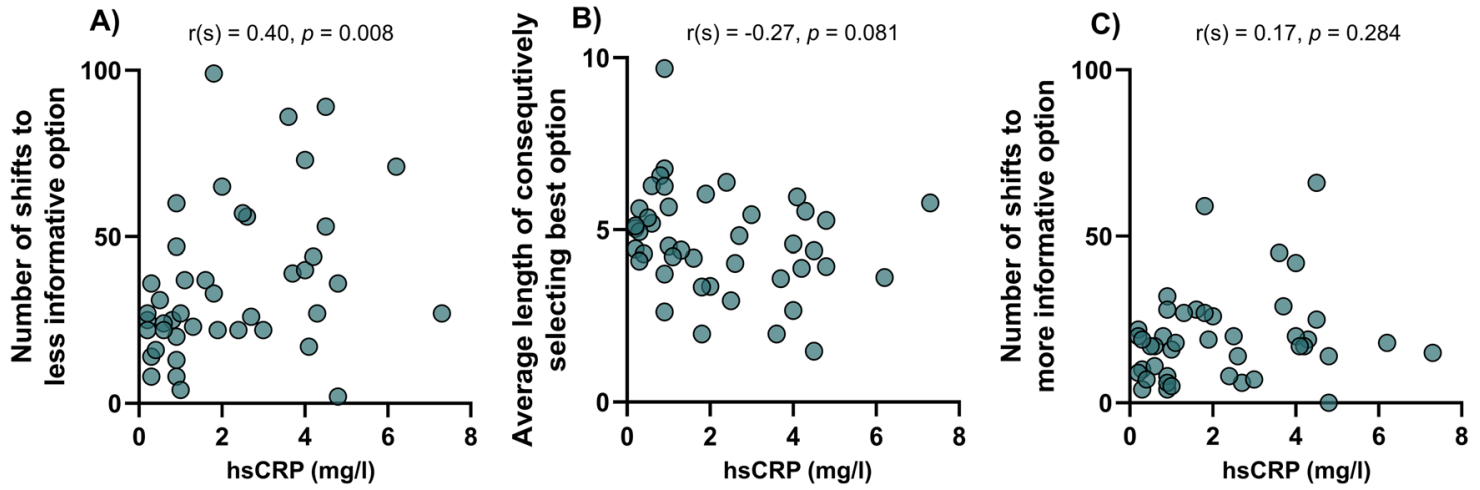

Fig. 3 Associations between task performance and high-sensitivity C-reactive protein in patients with schizophrenia. Within patients with SZ, correlation of hsCRP with A random exploration was significant, in contrast to correlation with B exploitation or C directed exploration.

can directly influence dopamine neurotransmission ${ }^{20}$. We therefore investigated if a pro-inflammatory state is associated with the observed task performance deficits and in extension the hypothesized hypodopaminergic state. In line with previous research ${ }^{25}$, patients with SZ showed a pro-inflammatory state. Across all the analyses investigating group differences of inflammatory parameters, CCL20, TRAIL, and hsCRP were most strongly associated with SZ group affiliation. In addition, hsCRP was positively correlated with random exploration, the task behavior that contributed to decreased overall task performance. Taken together, the current data suggest that patients with SZ show increased proinflammatory markers compared to $\mathrm{HC}$, and that hsCRP is associated with increased inefficient, random exploration.

At present, we cannot be certain about the exact mechanisms of how CRP causally affects exploratory behavior, because of the numerous functions of this acute-phase protein in the body's immune response ${ }^{41}$. Its production predominantly takes place in hepatocytes where it is induced by pro-inflammatory cytokines, mainly IL-6 and TNF-a (ref. ${ }^{42}$ ). While CRP is widely used in clinical practice to detect and monitor the course of a variety of inflammatory and immune processes, much less is known about its function ${ }^{43}$. One of the major roles of CRP is the activation of the classical complement pathway ${ }^{44}$. In circulation, the complement system interacts with pathogens to mark them for destruction by phagocytes ${ }^{45}$. The literature regarding findings in patients with SZ is inconclusive: while some studies have shown increased peripheral complement protein concentration in patients with $\mathrm{SZ}^{46,47}$, a recent meta-analysis has concluded that the evidence regarding complement system dysregulation is mixed ${ }^{48}$. In addition, recent evidence has suggested that the complement cascade is involved in several central nervous system processes, including neurogenesis and synaptic pruning, and has specifically been implicated in $\mathrm{SZ}^{49,50}$. Further studies are needed to investigate how peripheral complement levels are linked to the central nervous system and pathologies relevant to SZ, especially given that complements do not cross an intact blood-brain barrier $(\mathrm{BBB})^{51}$. An additional potential role of CRP with relevance to SZ could be its ability to facilitate recruitment of peripheral leukocytes to the brain. Recent studies have indicated some evidence that a subgroup of patients with SZ display signs of 
leukocyte infiltration into the prefrontal cortex ${ }^{52}$. In vitro studies have shown that CRP promotes CCL2 mediated chemotaxis through the upregulation of chemokine receptor (CCR)2 on monocytes ${ }^{53}$. Although preliminary, this could constitute an interesting mechanism. While CRP itself does not cross the BBB, peripheral CRP was shown to directly increase the permeability of the BBB by reducing tight junction proteins ${ }^{54}$. This increased BBB permeability could facilitate the access of potentially neurotoxic substances, such as pro-inflammatory cytokines, peripheral leukocytes, or autoantibodies to the brain ${ }^{25,55}$.

In contrast to CRP, we did not find an association between TRAIL or CCL20, and exploratory behavior although they were increased in patients with SZ compared to HC. TRAIL (or tumor necrosis factor (ligand) superfamily, member 10 (TNFSF10)) is a member of the TNF cytokine superfamily ${ }^{56}$. One of the major functions of TRAIL is the induction of apoptosis ${ }^{57,58}$. Since apoptotic processes of neurons have been implicated in the pathophysiology of $\mathrm{SZ}^{59}$, TRAIL induced neuronal apoptosis could constitute an interesting potential disease mechanism. However, further studies investigating a causal mechanism are warranted. Chemokine ( $\mathrm{C}-\mathrm{C}$ motif) ligand 20 (CCL20), also known as macrophage inflammatory protein $3 a$, is expressed in various human tissues and immune cells ${ }^{60}$ and is the only chemokine ligand to the chemokine receptor $\mathrm{CCR6}^{61}$. Multiple immune cells express CCR6, including both proinflammatory Th17 and regulatory $T$ cells ${ }^{62}$. Dysregulation of peripheral T cells has been associated with SZ and CCL20 could potentially play a role in this dysregulation, but direct evidence for this link still needs to be elucidated ${ }^{63-67}$.

There are several limitations that should be considered when interpreting the results of the current study: first, patients were medicated, and although a recent meta-analysis has not found an effect of antipsychotics on $\mathrm{CRP}^{25}$, smaller studies have shown associations between antipsychotic medication and $\mathrm{CRP}^{68}$, so a confounding effect of certain classes of antipsychotics on both behavior and metabolism cannot be excluded. Moreover, to causally test the hypothesis that peripheral inflammatory mediators can indeed influence neurotransmitter levels leading to behavioral changes, future studies directly measuring dopamine and pharmacologically blocking dopamine receptors in combination with experimentally induced immune challenges in $\mathrm{HC}$, patients with SZ, and preclinical models are needed.

Taken together, the present study suggests that patients with SZ are more likely to randomly explore than HC and that this behavior is associated with symptoms of disorganization. Importantly, this inefficient form of exploratory behavior is associated with a pro-inflammatory state. Further studies are needed to elucidate the underlying mechanism of how CRP leads to the neurobiological changes resulting in the observed behavioral alterations. Nevertheless, our data suggest that hsCRP could be a marker for the severity and treatment course of and potentially a therapeutic target for maladaptive exploratory behaviors in SZ, and potentially other neuropsychiatric disorders.

\section{METHODS \\ Participants}

Forty five patients (24 outpatients and 21 inpatients) meeting the DSM-V (ref. ${ }^{69}$ ) criteria for SZ and $19 \mathrm{HC}$ subjects were recruited. Patients were recruited from outpatient and inpatient units of the Psychiatric Hospital of the University of Zurich and affiliated institutions, HC were recruited from the community via advertisement. More patients than controls were recruited to have adequate power for the correlational analyses (see below). Two patients did not complete the bandit task (due to fatigue) and are therefore only included in the group comparison of immune markers. Diagnoses were confirmed by conducting the Mini-International Neuropsychiatric Interview ${ }^{70}$. All patients were clinically stable and under a stable dose of medication for at least 2 weeks prior to testing. Inpatients were at the end of their hospitalization, and engaged in a multimodal therapy program and activities outside the hospital. The average duration of hospitalization for patients with SZ in Swiss psychiatric hospitals is longer than in most other countries, so the majority of patients would have been treated as outpatients in other health care systems $\mathrm{s}^{71,72}$. The inclusion age was between 18-65 years. We excluded patients with any other than the above mentioned DSM-IV Axis I disorder (including major depressive disorders), benzodiazepines (except lorazepam equivalents of $1 \mathrm{mg}$ or less per day), and acute psychotic symptoms. Participants with any alcohol use disorder based on lifetime criteria and participants with current cannabis abuse or dependency, or with any other substance abuse were excluded. Chlorpromazine equivalents were calculated according to ref. ${ }^{73}$. $\mathrm{HC}$ were excluded if any neuropsychiatric diagnosis was present in the structured Mini-International Neuropsychiatric Interview. In both groups, participants were excluded if they had a history of head injury or any autoimmune or chronic inflammatory disorder, or if they took any pain medication or antiinflammatory drugs at least 1 week prior to testing (assessed by detailed questionnaire and medical records where available). Furthermore, participants were not included in the study if they had a history of any known acute inflammation 2 weeks prior to testing. All participants gave written informed consent and the project was approved by the Ethics Committee of the Canton of Zurich.

\section{Assessment of psychopathology and cognition}

On the day of testing, study participants were first screened for the presence of symptoms of an acute infection and their fasting state was verified by a questionnaire. After the blood draw (see below), their diagnosis was confirmed with the Mini-International Neuropsychiatric Interview ${ }^{70}$. Then, psychopathological symptoms were assessed with the PANSS ${ }^{74}$. The PANSS factor scores were calculated according to the five-factor model of Wallwork and colleagues ${ }^{75}$ The negative symptom factor was calculated based on items N1, N2, N3, N4, N6, G7, the positive factor computed from items P1, P3, P5, G9, the disorganized factor was calculated based on items P2, N5, G11, and the depressive factor computed from items P4, P7, G8, and G14. Cognition was assessed with the Brief Neurocognitive Assessment (BNA) ${ }^{76}$, which was shown to be highly correlated with the MATRICS Consensus Cognitive Battery ${ }^{77}$ and has similarly good psychometric properties ${ }^{78}$. With the BNA, a cognitive score is computed for each participant by combining results from the Letter-Number span (sequencing) (LNS) test and the Symbol Coding Test (SCT) ${ }^{76}$. In the LNS, participants are read a sequence of letters and digits which they then have to report back, listing first the numbers in ascending order, and then the letters in alphabetical order ${ }^{78}$. This test was shown to measure working memory capacity and attention ${ }^{79}$. The SCT allows to measure processing speed; participants are required to assign in a given time as many numbers from an item-number key to the corresponding item ${ }^{78}$. Results of the subtests were then $z$-transformed based on the data of the $\mathrm{HC}$ group and a composite cognitive score was computed as the mean of the $z$-transformed test scores. The global level of functioning was assessed using the PSP ${ }^{80}$.

\section{Bandit task}

For assessing exploratory vs. exploitative behavior, the participants performed a computerized virtual slot machine (bandit) task (Supplementary Fig. 1). The procedure is an adapted version of a recently described bandit task ${ }^{14}$. Briefly, participants were shown three virtual slot machines depicted by three blue boxes on a screen between which they had to choose repeatedly by pressing the arrow keys on the keyboard. After each choice, the points they won, ranging from 1 to 100 , were displayed. Participants were instructed to collect as many points as possible to maximize their gain. In foraging situations, like in this bandit task, decision makers need to balance exploitation with exploration. They were informed that the points paid out by the slot machines oscillated randomly and independently over trials, so they would have to choose at any given point between exploiting the currently used bandit and exploring the other two options to maximize gain. Unlike standard slots, the mean payoffs changed randomly and independently from trial to trial, with subjects finding information about the current worth of a slot only through sampling it ${ }^{13}$. Three brief written questions and a short practice trial to confirm the comprehension of the task took place before the actual task began. The payouts for each bandit were generated with a decaying Gaussian random walk, resulting in points paid off varying noisily around three different means. Reward of the selected option was displayed on the computer screen after each choice. If participants took too much time $(5 \mathrm{~s})$ deciding between the slot machines, a window popped up asking them to choose a machine. Participants completed 200 trials. At the end, five trials were 
randomly selected and the participants received the mean of the corresponding gains. To test whether participants performed better than chance, we simulated random choosers (equal chance to select each bandit) 1000 times for each participant's reward structure. The average total reward earned by the random choosers was 51.90 points. We tested whether control participants and participants with SZ performed better or worse than the average of random choosers. To avoid any unnecessary assumptions about the actual implementation of psychological functions, we performed model-free analyses. We defined the best, second-best and worst options as the options with the highest, second highest, and lowest expected value in the current trial based on the objective reward structure of the task. Continuously selecting the best option was defined as exploitation. There was a significant correlation between the average length of consecutively selecting the best option and points earned in the task $(r(s)=0.53, p<0.001)$, further validating our measure of exploitation. Because the points paid out by the slot machines oscillated randomly and independently over trials, too strong exploitation with result in nonoptimal behavior, where people have insufficient information about the current reward structure. Ideally, decision makers should balance exploitation with exploration. To further disentangle different forms of exploratory behaviors, we distinguished directed from random exploration based on the uncertainty associated with each option ${ }^{36,81,82}$. The less often a given bandit has been chosen, the less information the decision maker has about the bandit. Accordingly, directed exploration corresponds to choosing the option that provides most information for future decisions, and can be viewed as goal-directed uncertainty reduction. In contrast, random exploration can be interpreted as a noisy behavior, which provides little or no extra information. In our task, directed exploration corresponds to a shift to the least selected option while shifts to more often selected options correspond to random exploration. A less well-established alternative definition of directed and random exploration is based on the value of each option, with value-based directed exploration defined as switching from the best option to the second-best option. In the supplemental material, we show that the main findings of the present study remained qualitatively the same using this alternative definition (Supplementary Fig. 2).

\section{Blood samples and processing}

Blood was drawn between 8 and 10 a.m. on the day of testing. Study participants were instructed to fast for at least $8 \mathrm{~h}$ prior to the blood draw and this was verified by a questionnaire on the day of testing. To obtain plasma for the Olink panel, blood was drawn into ethylenediaminetetraacetic acid tubes (Sarstedt, Switzerland), centrifuged for $15 \mathrm{~min}$ at $1500 \times g$ and plasma was frozen at $-80^{\circ} \mathrm{C}$. hsCRP was measured in serum: blood was collected into a silica and gel containing tube (BD Vacutainer).

\section{hsCRP}

hsCRP was measured in participant serum samples by immunoturbidimetry on Abbott Architect c16000 or c8000, at Unilabs Medical Analytics in Duebendorf/Zurich, Switzerland.

\section{Cytokines and chemokines}

Proteins were measured using the Olink $^{\circledR}$ Inflammation panel (Olink Proteomics AB, Uppsala, Sweden) according to the manufacturer's instructions. The Proximity Extension Assay technology used for the Olink protocol is described in detail in ref. ${ }^{83}$. In brief, pairs of oligonucleotidelabeled antibody probes bind to their targeted protein, and if the two probes are brought in close proximity the oligonucleotides will hybridize in a pair-wise manner. The addition of a DNA polymerase leads to a proximity-dependent DNA polymerization event, generating a unique PCR target sequence. The resulting DNA sequence is subsequently detected and quantified using a microfluidic real-time PCR instrument (Biomark HD, Fluidigm). Data are then quality-controlled and normalized using an internal extension control and an inter-plate control, to adjust for intraand inter-run variation. The final assay readout is presented in normalized protein expression values, which is an arbitrary unit on a log2-scale, where a high value corresponds to a higher protein expression. Detection limits are available on the manufacturer's website (www.olink.com). In total, 91 proteins were measured with an intra- and inter-assay coefficient of variance of $<10 \%$. We excluded 18 proteins due to $>80 \%$ values below the limit of detection (LOD). Values below the LOD were replaced with the LOD for the specific assay. Five participants (two HC and three SZ) were excluded because their samples failed quality control.

\section{Data analysis}

All variables were tested for normal distribution using the Shapiro-Wilk test. If variables were normally distributed, potential group differences were examined using two-sided Students $t$ tests. To investigate correlations between inflammatory parameters and task variables/psychopathology, Pearson correlation coefficients $r(p)$ were calculated. If data were not normally distributed, we instead used Mann-Whitney $U$ tests and Spearman correlation coefficients $r(s)$. Heat maps of inflammatory markers are based on standardized scores that were calculated as: (value of SZ (individual sample value) - mean of HCs)/standard deviation of HCs). The level of significance was set at $p<0.05$. To investigate group differences in inflammatory markers between $\mathrm{HC}$ and patients with $\mathrm{SZ}$, we used an exploratory approach using uncorrected test statistics and network analysis (see below). Correlations between immune markers and task behavior was controlled for multiple comparisons using the procedure proposed by Benjamini and Hochberg ${ }^{84}$. General linear models were used to test for the effects of covariates on group differences and partial correlations were calculated when controlling for covariates in the correlational analyses. Effect size was assessed by calculating Cohen's $d$. Statistical analyses were computed with SPSS version 25 (IBM Corp., SPSS Inc., Chicago IL, USA), Stata version 14.2 (StataCorp. 2015. Stata Statistical Software: Release 14. College Station, TX: StataCorp LP), Matlab version R2018a (The MathWorks, Inc., Natick, Massachusetts, United States), R (Version 3.4.0 (ref. ${ }^{85}$ ), and GraphPad Prism software (GraphPad Software Inc.). Heat maps were created using the online tool Morpheus (https:// software.broadinstitute.org/morpheus).

\section{Network analysis}

Network analyses followed a four-step procedure: (i) data preparation: all variables for which the $t$ test for differences in blood levels of investigated inflammation markers between patients with $\mathrm{SZ}$ and $\mathrm{HC}$ had a $p$ value $<0.05$ before correction for multiple testing were included in the network. (ii) Network estimation and visualization: the estimated network is a Gaussian Graphical Model, in which the nodes represent the included variables. Edges between these variables can be interpreted as undirected, weighted partial correlations. Partial correlations can be thought of as the "unique" correlation between two nodes after controlling for the correlation with all other nodes ${ }^{86}$. We then visualized the network, with the thickness of an edge corresponding to its weight and with the color indicating a positive (red) or negative (blue) partial correlation between two nodes. Placement of the nodes for the visualization was based on the Fruchterman-Reingold algorithm ${ }^{87}$. (iii) Network characterization: we estimated centrality indices, which are a relative marker for the influence of a node on the network as a whole (for detail see ref. ${ }^{88}$ ). (iv) Network accuracy and stability: using current state of the art procedures, we calculated $95 \%$ confidence intervals for the edge weights and difference test for centrality and edge weights. All network analyses were carried out in R (version 3.6.1 (ref. ${ }^{85}$ )) using the package bootnet ${ }^{86}$. Networks were visualized using qgraph ${ }^{89}$. Detailed information about network analysis are provided in the SI.

\section{Reporting summary}

Further information on experimental design is available in the Nature Research Reporting Summary linked to this paper.

\section{DATA AVAILABILITY}

The data that support the findings of this study are available upon reasonable request from the corresponding author.

Received: 29 June 2020; Accepted: 24 November 2020; Published online: 03 February 2021

\section{REFERENCES}

1. Kahn, R. S. et al. Schizophrenia. Nat. Rev. Dis. Prim. 1, 15067 (2015).

2. Rocca, P. et al. Disorganization and real-world functioning in schizophrenia: results from the multicenter study of the Italian Network for Research on Psychoses. Schizophr. Res. 201, 105-112 (2018).

3. Insel, T. R. Rethinking schizophrenia. Nature 468, 187-193 (2010).

4. Foussias, G. et al. Prediction of longitudinal functional outcomes in schizophrenia: the impact of baseline motivational deficits. Schizophrenia Res. 132, 24-27 (2011). 
5. Strauss, G. P. et al. Deconstructing negative symptoms of schizophrenia: avolition-apathy and diminished expression clusters predict clinical presentation and functional outcome. J. Psychiatr. Res. 47, 783-790 (2013).

6. Brown, R. G. \& Pluck, G. Negative symptoms: the 'pathology' of motivation and goal-directed behaviour. Trends Neurosci. 23, 412-417 (2000).

7. Sitnikova, T., Goff, D. \& Kuperberg, G. R. Neurocognitive abnormalities during comprehension of real-world goal-directed behaviors in schizophrenia. J. Abnorm. Psychol. 118, 256-277 (2009).

8. Luther, L., Fischer, M. W., Firmin, R. L. \& Salyers, M. P. Clarifying the overlap between motivation and negative symptom measures in schizophrenia research: a meta-analysis. Schizophr. Res. 206, 27-36 (2019).

9. Siddiqui, I. et al. Objective assessment of exploratory behaviour in schizophrenia using wireless motion capture. Schizophr. Res. 195, 122-129 (2018).

10. Addicott, M. A., Pearson, J. M., Sweitzer, M. M., Barack, D. L. \& Platt, M. L. A primer on foraging and the explore/exploit trade-off for psychiatry research. Neuropsychopharmacology 42, 1931-1939 (2017).

11. Pratt, S. C. \& Sumpter, D. J. A tunable algorithm for collective decision-making. Proc. Natl Acad. Sci. USA 103, 15906-15910 (2006).

12. Gittins, J. C. Bandit processes and dynamic allocation indices. J. R. Stat. Soc. Ser. B (Methodol.) 41, 148-177 (1979).

13. Daw, N. D., O'Doherty, J. P., Dayan, P., Seymour, B. \& Dolan, R. J. Cortical substrates for exploratory decisions in humans. Nature 441, 876-879 (2006).

14. Raja Beharelle, A., Polania, R., Hare, T. A. \& Ruff, C. C. Transcranial stimulation over frontopolar cortex elucidates the choice attributes and neural mechanisms used to resolve exploration-exploitation trade-offs. J. Neurosci. 35, 14544-14556 (2015).

15. Cohen, J. D., McClure, S. M. \& Yu, A. J. Should I stay or should I go? How the human brain manages the trade-off between exploitation and exploration. Philos. Trans. R. Soc. Lond. B Biol. Sci. 362, 933-942 (2007).

16. Strauss, G. P. et al. Deficits in positive reinforcement learning and uncertaintydriven exploration are associated with distinct aspects of negative symptoms in schizophrenia. Biol. Psychiatry 69, 424-431 (2011).

17. Martinelli, C., Rigoli, F., Averbeck, B. \& Shergill, S. S. The value of novelty in schizophrenia. Schizophr. Res. 192, 287-293 (2018).

18. Yu, A. J. \& Dayan, P. Uncertainty, neuromodulation, and attention. Neuron 46, 681-692 (2005).

19. Aston-Jones, G. \& Cohen, J. D. Adaptive gain and the role of the locus coeruleusnorepinephrine system in optimal performance. J. Comp. Neurol. 493, 99-110 (2005).

20. Treadway, M. T., Cooper, J. A. \& Miller, A. H. Can't or won't? Immunometabolic constraints on dopaminergic drive. Trends Cogn. Sci. 23, 435-448 (2019).

21. Felger, J. C. \& Treadway, M. T. Inflammation effects on motivation and motor activity: role of dopamine. Neuropsychopharmacology 42, 216-241 (2017).

22. Dantzer, R. Neuroimmune interactions: from the brain to the immune system and vice versa. Physiol. Rev. 98, 477-504 (2018).

23. Cathomas, F., Murrough, J. W., Nestler, E. J., Han, M. H. \& Russo, S. J. Neurobiology of resilience: interface between mind and body. Biol. Psychiatry 86, 410-420 (2019).

24. Miller, B. J., Buckley, P., Seabolt, W., Mellor, A. \& Kirkpatrick, B. Meta-analysis of cytokine alterations in schizophrenia: clinical status and antipsychotic effects. Biol. Psychiatry 70, 663-671 (2011).

25. Fernandes, B. S. et al. C-reactive protein is increased in schizophrenia but is not altered by antipsychotics: meta-analysis and implications. Mol. Psychiatry 21, 554-564 (2016).

26. Dantzer, R. Cytokine, sickness behavior, and depression. Immunol. Allergy Clin. North Am. 29, 247-264 (2009).

27. Maes, M. et al. Depression and sickness behavior are Janus-faced responses to shared inflammatory pathways. BMC Med. 10, 66 (2012).

28. Miller, A. H. \& Raison, C. L. The role of inflammation in depression: from evolutionary imperative to modern treatment target. Nat. Rev. Immunol. 16, 22-34 (2016).

29. Culbreth, A. J., Gold, J. M., Cools, R. \& Barch, D. M. Impaired activation in cognitive control regions predicts reversal learning in schizophrenia. Schizophr. Bull. 42, 484-493 (2016).

30. Reddy, L. F., Waltz, J. A., Green, M. F., Wynn, J. K. \& Horan, W. P. Probabilistic reversal learning in schizophrenia: stability of deficits and potential causal mechanisms. Schizophr. Bull. 42, 942-951 (2016).

31. Ventura, J., Thames, A. D., Wood, R. C., Guzik, L. H. \& Hellemann, G. S. Disorganization and reality distortion in schizophrenia: a meta-analysis of the relationship between positive symptoms and neurocognitive deficits. Schizophr. Res. 121, 1-14 (2010).

32. Monteiro, L. C., Silva, V. A. \& Louza, M. R. Insight, cognitive dysfunction and symptomatology in schizophrenia. Eur. Arch. Psychiatry Clin. Neurosci. 258, 402-405 (2008).

33. Sigaudo, M. et al. Quality of life in stable schizophrenia: the relative contributions of disorganization and cognitive dysfunction. Schizophr. Res. 153, 196-203 (2014).
34. Evans, J. D. et al. Cognitive and clinical predictors of success in vocational rehabilitation in schizophrenia. Schizophr. Res. 70, 331-342 (2004).

35. Minor, K. S. \& Lysaker, P. H. Necessary, but not sufficient: links between neurocognition, social cognition, and metacognition in schizophrenia are moderated by disorganized symptoms. Schizophr. Res. 159, 198-204 (2014).

36. Frank, M. J., Doll, B. B., Oas-Terpstra, J. \& Moreno, F. Prefrontal and striatal dopaminergic genes predict individual differences in exploration and exploitation. Nat. Neurosci. 12, 1062-1068 (2009).

37. Gershman, S. J. \& Uchida, N. Believing in dopamine. Nat. Rev. Neurosci. 20, 703-714 (2019).

38. Humphries, M., Khamassi, M. \& Gurney, K. Dopaminergic control of the exploration-exploitation trade-off via the basal ganglia. Front. Neurosci. 6, 9 (2012).

39. Miller, B. J., Culpepper, N. \& Rapaport, M. H. C-reactive protein levels in schizophrenia: a review and meta-analysis. Clin. Schizophr. Relat. Psychoses 7, 223-230 (2014).

40. Fond, G. et al. Chronic low-grade peripheral inflammation is associated with ultra resistant schizophrenia. Results from the FACE-SZ cohort. Eur. Arch. Psychiatry Clin. Neurosci. 269, 985-992 (2019).

41. Tillett, W. S. \& Francis, T. Serological reactions in pneumonia with a non-protein somatic fraction of pneumococcus. J. Exp. Med. 52, 561-571 (1930).

42. Sproston, N. R. \& Ashworth, J. J. Role of C-reactive protein at sites of inflammation and infection. Front. Immunol. 9, 754 (2018).

43. Luan, Y.-Y. \& Yao, Y.-M. The clinical significance and potential role of C-reactive protein in chronic inflammatory and neurodegenerative diseases. Front. Immunol. 9, 1302-1302 (2018).

44. Kaplan, M. H. \& Volanakis, J. E. Interaction of C-reactive protein complexes with the complement system. I. Consumption of human complement associated with the reaction of C-reactive protein with pneumococcal C-polysaccharide and with the choline phosphatides, lecithin and sphingomyelin. J. Immunol. 112, 2135-2147 (1974).

45. Brown, J. S. et al. The classical pathway is the dominant complement pathway required for innate immunity to Streptococcus pneumoniae infection in mice. Proc. Natl Acad. Sci. USA 99, 16969-16974 (2002).

46. Santos Sória, L., Moura Gubert, C., Ceresér, K. M., Gama, C. S. \& Kapczinski, F. Increased serum levels of $\mathrm{C} 3$ and C4 in patients with schizophrenia compared to eutymic patients with bipolar disorder and healthy. Rev. Bras. Psiquiatr. 34, 119-120 (2012).

47. Laskaris, L. et al. Investigation of peripheral complement factors across stages of psychosis. Schizophr. Res. 204, 30-37 (2019).

48. Mongan, D. et al. Peripheral complement proteins in schizophrenia: a systematic review and meta-analysis of serological studies. Schizophr Res. 222, 58-72, https://doi.org/10.1016/j.schres.2020.05.036 (2020).

49. Stevens, B. et al. The classical complement cascade mediates CNS synapse elimination. Cell 131, 1164-1178 (2007).

50. Sekar, A. et al. Schizophrenia risk from complex variation of complement component 4. Nature 530, 177-183 (2016).

51. Morgan, B. P. Complement in the pathogenesis of Alzheimer's disease. Semin. Immunopathol. 40, 113-124 (2018).

52. Cai, H. Q. et al. Increased macrophages and changed brain endothelial cell gene expression in the frontal cortex of people with schizophrenia displaying inflammation. Mol. Psychiatry 25, 761-775 (2020).

53. Han, K. H. et al. C-reactive protein promotes monocyte chemoattractant protein1-mediated chemotaxis through upregulating CC chemokine receptor 2 expression in human monocytes. Circulation 109, 2566-2571 (2004).

54. Elwood, E., Lim, Z., Naveed, H. \& Galea, I. The effect of systemic inflammation on human brain barrier function. Brain Behav. Immun. 62, 35-40 (2017).

55. Campbell, B. M., Charych, E., Lee, A. W. \& Moller, T. Kynurenines in CNS disease: regulation by inflammatory cytokines. Front. Neurosci. 8, 12 (2014).

56. de Miguel, D., Lemke, J., Anel, A., Walczak, H. \& Martinez-Lostao, L. Onto better TRAILs for cancer treatment. Cell Death Differ. 23, 733-747 (2016).

57. Pan, G. et al. The receptor for the cytotoxic ligand TRAIL. Science 276, 111-113 (1997).

58. MacFarlane, M. et al. Identification and molecular cloning of two novel receptors for the cytotoxic ligand TRAIL. J. Biol. Chem. 272, 25417-25420 (1997).

59. Jarskog, L. F., Glantz, L. A., Gilmore, J. H. \& Lieberman, J. A. Apoptotic mechanisms in the pathophysiology of schizophrenia. Prog. Neuro-Psychopharmacol. Biol. Psychiatry 29, 846-858 (2005).

60. Power, C. A. et al. Cloning and characterization of a specific receptor for the novel CC chemokine MIP-3alpha from lung dendritic cells. J. Exp. Med. 186, 825-835 (1997).

61. Baba, M. et al. Identification of CCR6, the specific receptor for a novel lymphocytedirected CC chemokine LARC. J. Biol. Chem. 272, 14893-14898 (1997).

62. Comerford, l. et al. An immune paradox: how can the same chemokine axis regulate both immune tolerance and activation?: CCR6/CCL20: a chemokine axis 
balancing immunological tolerance and inflammation in autoimmune disease. BioEssays 32, 1067-1076 (2010).

63. Debnath, M. Adaptive immunity in schizophrenia: functional implications of T cells in the etiology, course and treatment. J. Neurolmmune Pharmacol. 10, 610-619 (2015).

64. Drexhage, R. C. et al. An activated set point of T-cell and monocyte inflammatory networks in recent-onset schizophrenia patients involves both pro- and antiinflammatory forces. Int. J. Neuropsychopharmacol. 14, 746-755 (2011).

65. Ding, M. et al. Activation of Th17 cells in drug naïve, first episode schizophrenia. Prog. Neuro-Psychopharmacol. Biol. Psychiatry 51, 78-82 (2014).

66. Fernandez-Egea, E. et al. Peripheral immune cell populations associated with cognitive deficits and negative symptoms of treatment-resistant schizophrenia. PloS ONE 11, e0155631 (2016).

67. Kelly, D. L. et al. Increased circulating regulatory T cells in medicated people with schizophrenia. Psychiatry Res. 269, 517-523 (2018)

68. Fond, G. et al. Relationships between low-grade peripheral inflammation and psychotropic drugs in schizophrenia: results from the national FACE-SZ cohort. Eur. Arch. Psychiatry Clin. Neurosci. 268, 541-553 (2018).

69. Association, A. P. Diagnostic and Statistical Manual of Mental Disorders: DSM-5. 5th edn, xliv, 947 (American Psychiatric Association, 2013).

70. Lecrubier, Y., Weiller, E. \& Herugeta, T. Mini International Neuropsychiatric Interview German Version 5.0. 0 (Psychiatrischen Universitätsklinik München, München 1999).

71. Kirschner, M. et al. Shared and dissociable features of apathy and reward system dysfunction in bipolar I disorder and schizophrenia. Psychological Med. 50, 936-947 (2020).

72. Hartmann, M. N. et al. Apathy but not diminished expression in schizophrenia is associated with discounting of monetary rewards by physical effort. Schizophr. Bull. 41, 503-512 (2015).

73. Woods, S. W. Chlorpromazine equivalent doses for the newer atypical antipsychotics. J. Clin. Psychiatry 64, 663-667 (2003).

74. Kay, S. R., Opler, L. A. \& Lindenmayer, J. P. The Positive and Negative Syndrome Scale (PANSS): rationale and standardisation. Br. J. Psychiatry Suppl. 155, 59-67 (1989).

75. Wallwork, R. S., Fortgang, R., Hashimoto, R., Weinberger, D. R. \& Dickinson, D. Searching for a consensus five-factor model of the Positive and Negative Syndrome Scale for schizophrenia. Schizophr. Res. 137, 246-250 (2012).

76. Fervaha, G., Agid, O., Foussias, G. \& Remington, G. Toward a more parsimonious assessment of neurocognition in schizophrenia: a 10-minute assessment tool. J. Psychiatr. Res. 52, 50-56 (2014).

77. Nuechterlein, K. H. et al. The MATRICS Consensus Cognitive Battery, part 1: test selection, reliability, and validity. Am. J. Psychiatry 165, 203-213 (2008).

78. Fervaha, G. et al. Examination of the validity of the Brief Neurocognitive Assessment (BNA) for schizophrenia. Schizophr. Res. 166, 304-309 (2015).

79. Crowe, S. F. Does the letter number sequencing task measure anything more than digit span? Assessment 7, 113-117 (2000).

80. Juckel, G. et al. Validation of the Personal and Social Performance (PSP) Scale in a German sample of acutely ill patients with schizophrenia. Schizophrenia Res. 104, 287-293 (2008).

81. Gershman, S. J. \& Tzovaras, B. G. Dopaminergic genes are associated with both directed and random exploration. Neuropsychologia 120, 97-104 (2018).

82. Somerville, L. H. et al. Charting the expansion of strategic exploratory behavior during adolescence. J. Exp. Psychol. Gen. 146, 155-164 (2017)

83. Assarsson, E. et al. Homogenous 96-plex PEA immunoassay exhibiting high sensitivity, specificity, and excellent scalability. PloS ONE 9, e95192 (2014).

84. Benjamini, Y. \& Hochberg, Y. Controlling the false discovery rate: a practical and powerful approach to multiple testing. J. R. Stat. Soc. Ser. B (Methodol.) 57, 289-300 (1995)

85. RCoreTeam. R: A Language and Environment for Statistical Computing [Internet]. R Foundation for Statistical Computing https://www.R-project.org (2017).

86. Epskamp, S., Borsboom, D. \& Fried, E. I. Estimating psychological networks and their accuracy: a tutorial paper. Behav. Res. Methods 50, 195-212 (2018).
87. Fruchterman, T. M. J. \& Reingold, E. M. Graph drawing by force-directed placement. Softw. Pract. Exp. 21, 1129-1164 (1991).

88. Opsahl, T., Agneessens, F. \& Skvoretz, J. Node centrality in weighted networks: generalizing degree and shortest paths. Soc. Netw. 32, 245-251 (2010).

89. Epskamp, S., Cramer, A. O. J., Waldorp, L. J., Schmittmann, V. D. \& Borsboom, D. qgraph: network visualizations of relationships in psychometric data. J. Stat. Softw. 48, 18 (2012).

\section{ACKNOWLEDGEMENTS}

This work was supported by a Postdoc.Mobility Fellowship of the Swiss National Science Foundation (F.C.), a Walter and Gertrud Siegenthaler Postdoctoral Fellowship (F.C.), and project support by the Hartmann Mueller Foundation (F.C.), Kurt und Senta Herrmann Stiftung (F.C.), and the Olga Mayenfisch Stiftung (M.H.). F.K. was supported by an Early.Postdoc Mobility Fellowship of the Swiss National Science Foundation. P. N.T. acknowledges support by the Swiss NSF (Grants PP00P1 150739 and 100014_165884). T.S. was supported by the Forschungskredit of the University of Zurich (FK-19-048). S.K. was funded by the Swiss National Science Foundation (10001CL_169783).

\section{AUTHOR CONTRIBUTIONS}

Study concept and design: F.C. and S.K. Acquisition, analysis, or interpretation of data: F.C., F.K., K.G., H.-K.C., A.R.B., T.S., R.S., M.N.H., and P.N.T. Manuscript writing: F.C., P.T., and S.K. Funding: F.C., M.N.H., E.S., and S.K. All authors edited and approved the final version of the manuscript.

\section{COMPETING INTERESTS}

The authors declare no competing interests.

\section{ADDITIONAL INFORMATION}

Supplementary information is available for this paper at https://doi.org/10.1038/ s41537-020-00133-0.

Correspondence and requests for materials should be addressed to F.C.

Reprints and permission information is available at http://www.nature.com/ reprints

Publisher's note Springer Nature remains neutral with regard to jurisdictional claims in published maps and institutional affiliations.

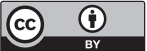

Open Access This article is licensed under a Creative Commons Attribution 4.0 International License, which permits use, sharing, adaptation, distribution and reproduction in any medium or format, as long as you give appropriate credit to the original author(s) and the source, provide a link to the Creative Commons license, and indicate if changes were made. The images or other third party material in this article are included in the article's Creative Commons license, unless indicated otherwise in a credit line to the material. If material is not included in the article's Creative Commons license and your intended use is not permitted by statutory regulation or exceeds the permitted use, you will need to obtain permission directly from the copyright holder. To view a copy of this license, visit http://creativecommons. org/licenses/by/4.0/.

(c) The Author(s) 2021 